Article

\title{
Effects of Variable Valve Lift on In-Cylinder Air Motion
}

\author{
Tianyou Wang ${ }^{1}$, Daming Liu ${ }^{2}$, Gangde Wang ${ }^{1}$, Bingqian Tan ${ }^{1}$ and Zhijun Peng ${ }^{1,3, *}$ \\ Received: 10 September 2015; Accepted: 11 November 2015; Published: 4 December 2015 \\ Academic Editor: Paul Stewart \\ 1 Stake Key Lab of Engines, Tianjin University, Tianjin 300072, China; wangtianyou@tju.edu.cn (T.W.); \\ wanggangde@tju.edu.cn (G.W.); tanbingqian@tju.edu.cn (B.T.) \\ 2 School of Automotive and Transportation, Tianjin University of Technology and Education, Tianjin 300222, \\ China; ldam@tju.edu.cn \\ 3 School of Engineering and Technology, University of Hertfordshire, Hatfield AL10 9AB, UK \\ * Correspondence: pengzj@tju.edu.cn; Tel.: +86-22-2740-6842
}

\begin{abstract}
An investigation into in-cylinder swirl and tumble flow characteristics with reduced maximum valve lifts (MVL) is presented. The experimental work was conducted in the modified four-valve optical spark-ignition (SI) test engine with three different MVL. Particle image velocimetry (PIV) was employed for measuring in-cylinder air motion and measurement results were analyzed for examining flow field, swirl and tumble ratio variation and fluctuating kinetic energy distribution. Results of ensemble-averaged flow fields show that reduced MVL could produce strong swirl flow velocity, then resulted in very regular swirl motion in the late stage of the intake process. The strong swirl flow can maintain very well until the late compression stage. The reduction of MVL can also increase both high-frequency and low-frequency swirl flow fluctuating kinetic energy remarkably. Regarding tumble flow, results demonstrate that lower MVLs result in more horizontal intake flow velocity vectors which can be easily detected under the valve seat area. Although the result of lower MVLs show a higher tumble ratio when the piston is close to the bottom dead centre (BDC), higher MVLs substantially produce higher tumble ratios which can be confirmed when most cylinder area lies in the measuring range.
\end{abstract}

Keywords: in-cylinder air motion; swirl flow; tumble flow; variable valve lift (VVL); particle image velocimetry (PIV)

\section{Introduction}

In recent years, advanced combustion technologies like gasoline direct injection (GDI) [1], gasoline CAI (controlled auto-ignition) [2], etc. have made significant contributions to improving the fuel economy of gasoline engines and for reducing $\mathrm{CO}_{2}$ emissions, in particular with support from variable valve actuation (VVA) [3,4] which has played a critical role in enhancing the combustion performance of gasoline engines. From recent production, in particular combined with downsizing, it has been seen that new GDI engines have achieved significant fuel consumption improvements over conventional port fuel injection (PFI) gasoline engines [5].

With regard to the application of VVA in gasoline engines, there are several possible control strategy options. Basically it can run as variable valve timing (VVT) and/or variable valve lift (VVL), though some VVA provides some different operation options, such as two valve-open pulses etc. [6]. As the most familiar VVA technology, VVT has been applied as the main valve phasing technology practically since the last decade for improving volumetric efficiency, for reducing the pumping losses and for reducing other harmful emissions. To achieve those benefits, early/late intake 
valve closing (EIVC/LIVC), late intake valve opening (LIVO), and variable valve overlap (VVO) have been implemented.

The main function of VVA is generally to replace the throttle under suitable operating conditions for regulating the intake air, thus achieving lower pumping losses than the throttle operation. Sometime, a VVL system can be integrated into appropriate cams with different profiles and corresponding maximum valve lift (MVL) for an optimal relationship between discharge coefficient and crank angle (CA) [7]. Compared to throttled operations, VVL with general valve timing and opening duration could help to maintain a higher in-cylinder pressure (or lower vacuum) during the early intake stage. Only during the late intake stage, lower valve lift will make the in-cylinder pressure be similar to the case with a throttle [8]. As demonstrated by Grimaldi et al. [9], the pumping loss of a non-throttled VVL configuration could be decreased approximately by $50 \%$ compared to that of throttled conditions. In recent years, VVL has been successfully applied on several models of production engines. Mitsubishi Motors Corporation developed a unique multi-mode valve system called mitsubishi innovative valve timing and lift electronic control (MIVEC) and has utilized it on a 4-cylinder in-line engine in early 1990s [10]. Another successful application of VVL on production engines is the new generation of BMW's Valvetronic system which is one real fully VVL system and through which the advantage of non-throttle load control can be completely realized [11]. Following those practical utilizations, new research and developments about flexible VVL are being continuously revealed, such as the twin mechanical variable lift (TMVL) valvetrain technology innovated by Ricardo Engineering Consultant Company [12] and 2-Step VVA developed by Delphi Corporation [13]. There are also other successful VVA technologies, such as those developed by Porsche, Audi, Fiat (UniAir and MultriAir), etc.

While VVL can reduce pumping losses for improving the energy efficiency of gasoline engines, its influence on in-cylinder air motion has been noted [14]. While a number of researches have demonstrated that the optimization of in-cylinder air motion is beneficial to the combustion and emission reduction, it is noted VVA might increase possibility of cycle-to-cycle variation $[15,16]$. Consequently to address this issue, relevant research using in-cylinder flow measurements have been carried out [17-19].

In an initial measurement on a steady flow test rig with the same cylinder head as used in the following investigation, results (as shown in Figure 1) have demonstrated that reduced MVL could significantly enhance in-cylinder swirl motion, but simultaneously reduce tumble motion strength. With those influences of VVL on in-cylinder flows, it will be necessary to investigate how air-fuel mixing and combustion would be affected under those conditions. In the present study, the swirl and tumble flow fields under reduced MVL have been examined with a particle image velocimetry (PIV) system and necessary data processing technology. Results concerning swirl ratio and tumble ratio, fluctuating kinetic energy of swirl and tumble flows and their high-frequency and low-frequency fractions have been analyzed to investigate the effects of VVL on the air-fuel mixing processing and combustion.

As most of current GDI engines have been equipped with turbochargers or supervisors, it is understood that the current research lacks necessary consideration of turbocharged condition. When the intake pressure is increased with possible boosters, the intake flow velocity will increase. Effects of reduced MVL on swirl and tumble flows might become more obvious. If this is correct, consequently the influence of reduced MVL on air-fuel mixing and combustion would be more important. One more factor which has been also ignored in the current research is the existence of the bowl shaped piston used for most GDI engines. Although it would become complicated with a bowl shaped piston, it would be more possible that swirl flows would be slowed down more or less and tumble flows could be accelerated if the bowl shape could be designed for this purpose. Meanwhile, while reduced MVL reduces the pumping losses and improves the air-fuel mixing and flame propagation, reduced swirl-tumble interaction as a result of the poor tumble flow might also affect the air-fuel mixing behavior. It has been reported in some research on diesel engines that air-fuel 
mixing is highly dependent on the interaction of swirl and tumble flows and the angle of spray [20]. Considering at least these three influences, it will be worthwhile to undertake a more detailed study in future investigations to examine the operating characteristics of GDI engines with reduced MVL.

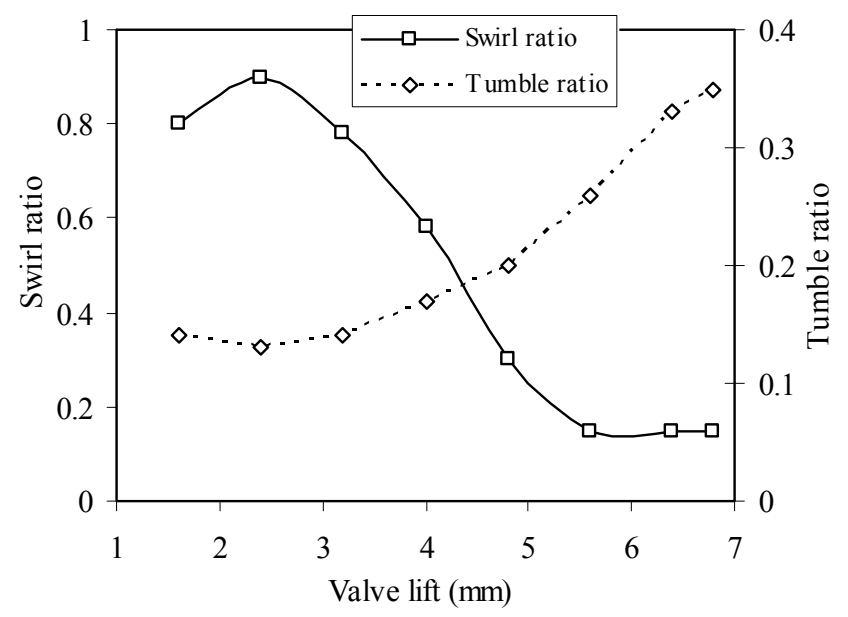

Figure 1. Influence of reduced maximum valve lifts (MVL) on the swirl ratio and tumble ratio under steady flow measurement.

\section{Experimental Section}

\subsection{Test Engine, Particle Image Velocimetry System and Experimental Conditions}

The optical engine employed for those PIV experiments is a modified Cagiva FOX350 four-valve single cylinder engine whose main specifications can be found in Table 1. A schematic including the optical engine system and the PIV system used for swirl and tumble flow measurement can be found in Figure 2. For measuring swirl and tumble flows, one extended transparent piston top with a $55 \mathrm{~mm}$ diameter quartz window and one $27 \mathrm{~mm}$ long quartz ring that was mounted between the cylinder head and the cylinder block are installed. A mirror was fixed at an angle of $45^{\circ}$ on the extended cylinder block for reflecting swirl flow images in the horizontal plane through the piston top window to the charge coupled device (CCD) camera.

Table 1. Main specifications of FOX350 optical engine and operating condition. Before bottom dead centre: BBDC; after top dead centre: ATDC; before top dead centre: BTDC; after bottom dead centre: ABDC; CA: crank angle.

\begin{tabular}{ccc}
\hline Specification & Unit & Value \\
\hline Valve number & & 4 \\
Bore $\times$ Stroke & $\mathrm{mm} \times \mathrm{mm}$ & $78.7 \times 66.0$ \\
Compression ratio & & $6.7: 1$ \\
Combustion chamber & & Pent-roof shape \\
Intake valve diameter & $\mathrm{mm}$ & 30.0 \\
Exhaust valve diameter & $\mathrm{mm}$ & 26.0 \\
Intake port & & Siamesed, tangent \\
Exhaust valve open & ${ }^{\circ} \mathrm{CA}$ & 74 BBDC \\
Exhaust valve close & ${ }^{\circ} \mathrm{CA}$ & 26 ATDC \\
Intake valve open & ${ }^{\circ} \mathrm{CA}$ & 22 BTDC \\
Intake valve close & ${ }^{\circ} \mathrm{CA}$ & 68 ABDC \\
Engine speed & rpm & 960, motored \\
Other engine operating conditions & & Wide open throttle, \\
& & naturally aspired \\
\hline
\end{tabular}

Most parts of the original cylinder head were kept, except that camshafts were changed for different valve lifts. The engine has a general pent-roof type combustion chamber and a siamesed 
but tangent intake port. For two intake valves, the valve seating width is about $1.5 \mathrm{~mm}$, which gives approximately $140 \mathrm{~mm}^{2}$ of valve seating area. The MVL of the original valvetrain was $6.8 \mathrm{~mm}$. For this research, two more camshafts with MVLs of $4.0 \mathrm{~mm}$ and $1.7 \mathrm{~mm}$ were prepared and used on the engine. Considering this investigation is focused on the influences of VVA on in-cylinder flows, those different camshafts still have the same valve timings/phasing as that of the original valve train. Compared to those VVA which has both variable lift and variable timing/phasing, this may reduce the possibility to reduce the pumping losses.

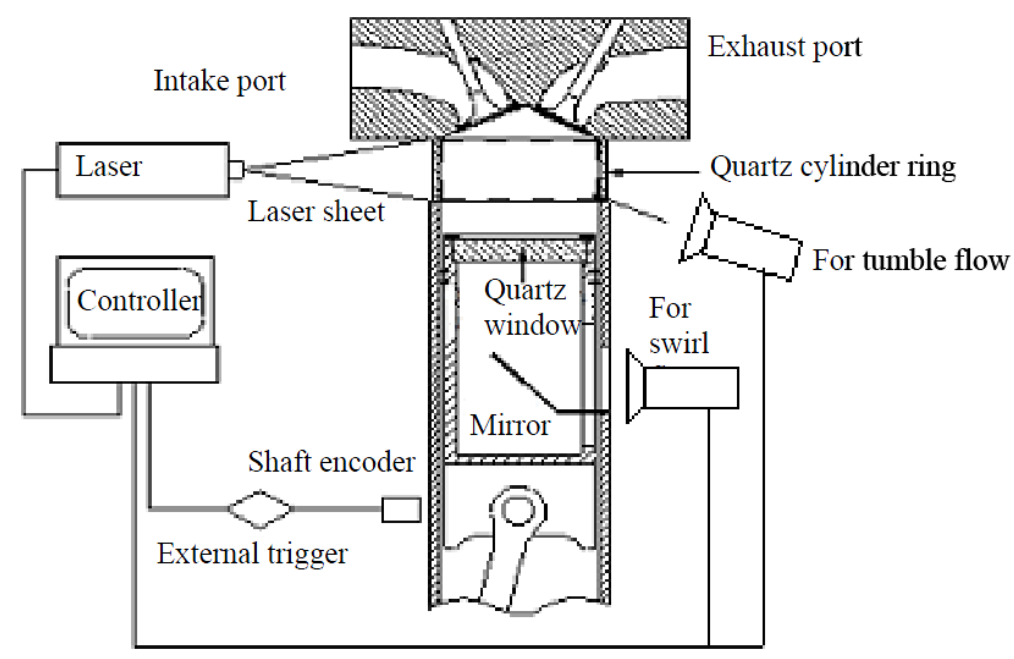

Figure 2. Optical engine set-up and particle image velocimetry (PIV) system for swirl flow and tumble flow measurements.

The valve profiles are presented in Figure 3, with $0{ }^{\circ} \mathrm{CA}$ referring to the top dead centre (TDC) at the beginning of the induction stroke. The engine speed is motored at $960 \mathrm{rpm}$, wide open throttle with naturally aspirated intake during all the experiments for different camshafts.

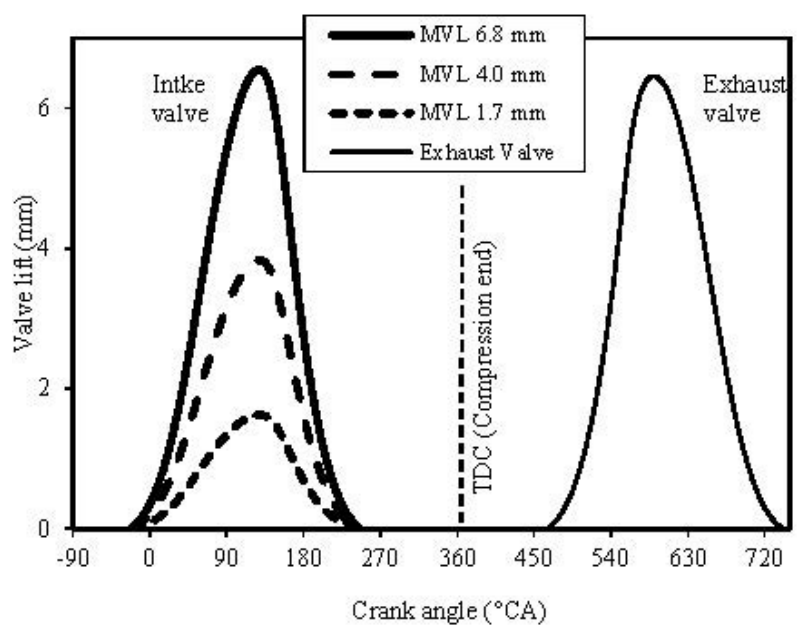

Figure 3. Valve lift profiles of variable valve lift (VVL).

The FlowMaster PIV system developed by LaVison [21] was employed for the flow field measurements. The basic setup has been presented in Figure 2. The light source is supplied by the second-harmonic output (532 nm) of two SOLO120 Nd:YAG lasers. Then, the laser beams were sent through a spherical lens (1000 $\mathrm{mm}$ focal length), followed by a concave cylindrical lens (-20 mm focal length). Finally the laser sheet with a thickness of about $2 \mathrm{~mm}$ at the beam waist was formed. 
The lasers have approximately $3-5 \mathrm{~ns}$ pulse width and $120 \mathrm{~mJ}$ pulse energy. The laser pulses from the two Nd:YAG lasers were controlled by the controller (shown in Figure 2) to fire at right CA and with a separation of $25 \mu \mathrm{s}$. A trigger signal was provided to the controller from a shaft encoder which has a $1{ }^{\circ} \mathrm{CA}$ resolution. A CCD camera (Sony ICX085 CCD sensor, Nikon AF Nikkor $50 \mathrm{~mm}$ f/1.8 lens, manufactured by Sony, Japan) with an image resolution of $1300 \times 1030$ and $6.7 \mu \mathrm{m}$ pixel spacing was used for image acquisition.

Seeding was added into the intake air flow by a Laskin sprayer (Filter Integrity Limited Company, Durham, UK) with disoctyl sebacate as solution. Air (3-5 bar) was supplied to the sprayer by an air compressor in accordance with the seeding density requirement. The particle mist had an average $1-3 \mu \mathrm{m}$ diameter.

When the in-cylinder swirl flows were investigated, the laser sheet was arranged horizontally to access the ring window. Then the images were obtained via the piston top window and the $45^{\circ}$ mirror, as shown in Figure 2. As the diameter of the optical window on the piston top is smaller than the cylinder bore, as shown in Figure 4a, as a result not all of the cross section area of the cylinder can be measured. Swirl flow images at three measuring planes of $5 \mathrm{~mm}, 10 \mathrm{~mm}$ and $15 \mathrm{~mm}$ horizontally under the TDC plane, respectively, were taken.

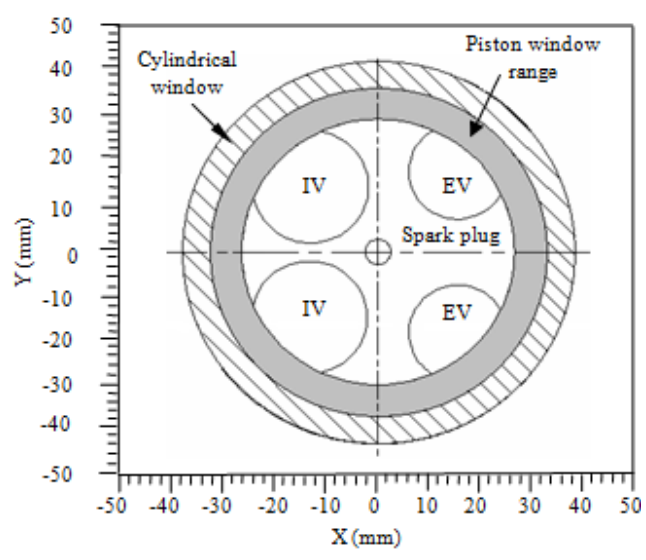

(a)

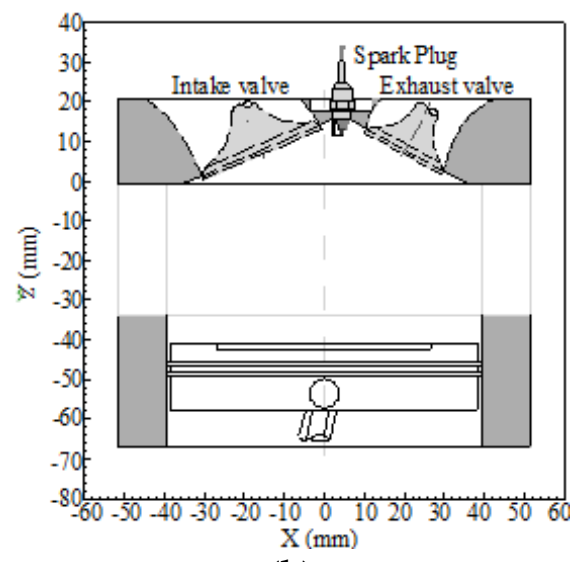

(b)

Figure 4. Optical measurement area for investigating (a) swirl flow and (b) tumble flow.

For exploring the tumble flows, the laser sheet did still access the ring window but in a vertical direction. The images were acquired through the ring window, by placing the CCD camera toward the laser sheet perpendicularly (as shown in Figure 2). Two measuring planes with one as the vertical symmetric plane in the cylinder (Plane 1 in Figure 5) and another through axes of same side intake and exhaust valves were selected for locating laser sheets. The tumble flow measurement area is shown in Figure $4 b$.

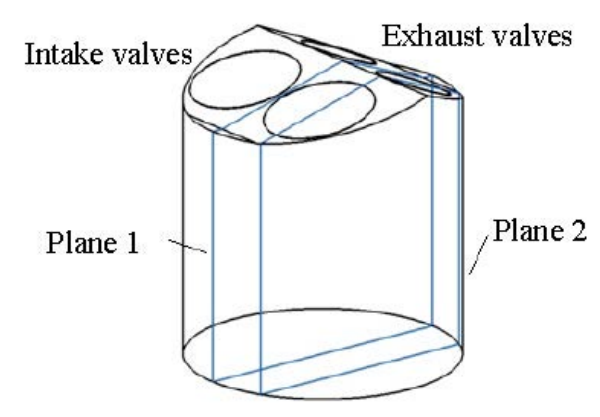

Figure 5. Optical measurement planes for investigating tumble flow. 
Synchronization with engine angular information and laser operation frequencies allowed having one measurement, i.e., one instantaneous velocity field, at each engine cycle. 100 consecutive cycles are performed at each given CA.

\subsection{Flow Parameters}

The in-cylinder flow field is highly turbulent during the individual cycles. Therefore, necessary analysis must be undertaken for identifying different flow parameters which might have different influence on flow performance. As most flow parameters and analysis technology have very similar application on both swirl and tumble flow, the definitions introduced in the following paragraphs will be based on swirl flow. When those are used on tumble flow analysis, one only needs to change the coordinates from $(x, y)$ (horizontal plane) to $(x, z)$ (vertical plane), as shown in Figure 4.

In order to identify the basic main flows, the cyclic variation and in-cycle turbulence levels, the instantaneous velocity vector $\vec{U}_{(x, y, \theta, i)}$ at the flow field in the horizontal planes with the coordinates of $(x, y)$ can be decomposed into an ensemble-averaged mean velocity vector and two fluctuating components:

$$
\vec{U}_{(x, y, \theta, i)}=\vec{U}_{\mathrm{EA}(x, y, \theta)}+\vec{u}_{\mathrm{LF}(x, y, \theta, i)}+\vec{u}_{\mathrm{HF}(x, y, \theta, i)}
$$

where, $\theta$ is the CA at which the measurement is performed and $i$ is the cycle number.

On the right hand side of Equation (1), the first term is the ensemble-average mean velocity over a number of cycles, as defined by Equation (2):

$$
\vec{U}_{\mathrm{EA}(x, y, \theta)}=\frac{1}{N} \sum_{i=1}^{N} \vec{U}_{(x \cdot y, \theta, i)}
$$

In the present study, the instantaneous results of 100 cycles were used for calculating the ensemble-average mean velocity. An example for one instantaneous flow field and the flow field of the ensemble-average mean velocity can be found in Figure $6 a, b$.

In Equation (1), the second $\vec{u}_{\mathrm{LF}(x, y, \theta, i)}$ and the third terms $\vec{u}_{\mathrm{HF}(x, y, \theta, i)}$ on the right hand side are related to the low-frequency velocity fluctuation and the high-frequency velocity fluctuation. For the measurement result of an instantaneous cycle, the sum of low-frequency and high-frequency fluctuations can be obtained by subtracting the ensemble-average mean velocity from the instantaneous velocity vector as defined in Equation (3).

For the in-cylinder flow in internal combustion engines, the low-frequency fluctuation normally refers to the cyclic variation but the high-frequency to the in-cycle turbulent velocity fluctuation. As the cyclic variation and the in-cycle velocity fluctuation have totally different impacts on the air-fuel mixing and consequent combustion process, it is necessary to extract them from the sum of fluctuations. In the present study, a low-pass filtering scheme in the spatial frequency domain which has been introduced by several researchers [22-24] was employed for that purpose. In order to identify an appropriate spatial cut-off frequency, at first the two-dimensional fluctuating velocity data were transformed into the spatial frequency domain using a two-dimensional fast Fourier transform (FFT). Then from the power spectral density (PSD) trace (as a function of spatial frequency), two higher PSD regions and an apparent low and flat PSD region between those two higher PSD regions could be found. Normally the spatial cut-off frequency could be determined by selecting a frequency value in the middle point of the low PSD region. In this study, the spatial cut-off frequency which was used is between $70 \mathrm{~m}^{-1}$ and $120 \mathrm{~m}^{-1}$, corresponding to $0.014 \mathrm{~m}$ and $0.008 \mathrm{~m}(14 \mathrm{~mm}$ and $8 \mathrm{~mm})$ in the spatial domain:

$$
{\overrightarrow{u_{F(x, y, \theta, i)}^{\prime}}}^{\prime}=\vec{U}_{(x, y, \theta, i)}-\vec{U}_{\mathrm{EA}(x, y, \theta)}
$$




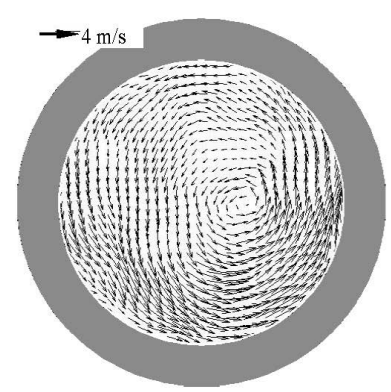

(a)

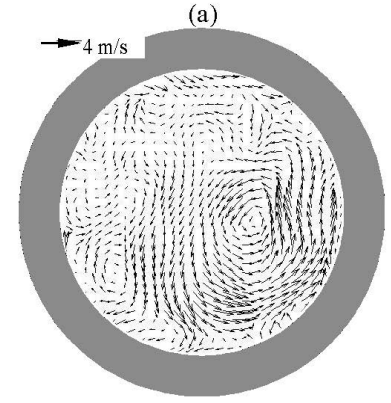

(c)

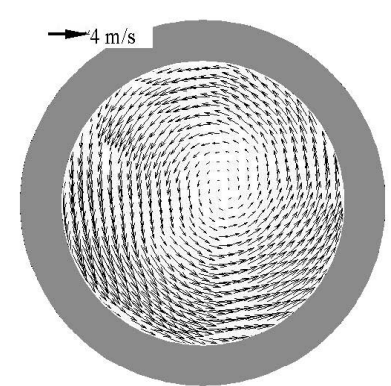

(b)

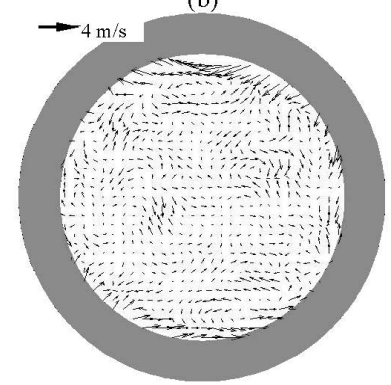

(d)

Figure 6. Swirl flow with ensemble-averaged velocity field and spatially filtered results for achieving high and low frequency fluctuations (for maximum valve lifts (MVL) $1.7 \mathrm{~mm}$ at the horizontal plane of $5 \mathrm{~mm}$ under dead centre (TDC)). (a) Instantaneous velocity filed; (b) ensemble-averaged mean velocity field; (c) low-frequency fluctuating velocity field; (d) high-frequency fluctuating velocity field.

Figure $6 c, d$ shows the flow fields of low-frequency and high-frequency fluctuating velocities estimated with the above method. After the fluctuating velocity components (low- or high frequency) were obtained, the ensemble-average root-mean-square (RMS) fluctuating velocity (for low frequency or high frequency) at the CA of $\theta$ could be calculated with the following formula:

$$
{\overrightarrow{u_{\mathrm{EA}}^{\prime}(x, y)}}^{\prime}=\sqrt{\left.\frac{1}{N} \sum_{i=1}^{N}{\overrightarrow{u_{F}^{\prime}}}_{F(x, y, i)}\right)^{2}}
$$

Similar to the estimate of the ensemble-average mean velocity, 100 cycles were still used for computing the ensemble-average RMS fluctuating velocity. Based on the above analysis, the kinetic energy of fluctuating velocity (for low-frequency or high-frequency) at $(x, y)$ could be derived as follows:

$$
E_{\mathrm{EA}(x, y)}=\frac{1}{2}\left(\left(u_{\mathrm{EA}(x, y)}^{\prime}\right)_{x}^{2}+\left(u_{\mathrm{EA}(x, y)}^{\prime}\right)_{y}^{2}\right)
$$

By integrating all kinetic energies of the fluctuating velocity at all spatial points of the measured plane, the total kinetic energy of fluctuating velocity at a measured plane could be estimated. Based on this, the total non-dimensional RMS fluctuating velocity can be obtained with the following definition:

$$
v_{\mathrm{RMS}}=\frac{\sqrt{2 E_{\mathrm{EA}(x, y)}}}{V_{\text {meanpiston }}}
$$

Finally, the swirl ratio $\left(R_{\mathrm{s}}\right)$ of a measured plane at the CA of $\theta$ could be derived by using Equation (7):

$$
R_{\mathrm{s}}=\frac{\sum_{j=1}^{M} m_{j} r_{j} U_{\mathrm{EA}(j, \theta)}}{\frac{2 \pi n}{60} \sum_{j=1}^{M} m_{j} r_{j}^{2}}
$$


where, $m_{j}$ is the mass of unit $j($ at $(x, y)) ; r_{j}$ is the distance from unit $j$ to the rotational axis of the flow; $U_{E A(j, \theta)}$ is the tangential component of velocity in $j ; n$ is the engine speed in rpm. To be noted, the coordinate system origin used here is the swirl center in the measured plane.

\section{Results and Discussion}

First of all, it needs to be noted that the CA is defined as that seen in Figure 3 for the results shown in the following sections, The TDC when the intake valve just opens has a CA of $0{ }^{\circ} \mathrm{CA}$ and the compression/combustion TDC is at $360^{\circ} \mathrm{CA}$.

\subsection{Effects of Maximum Valve Lifts on Swirl Flow Field and Swirl Ratio}

As shown in Figure 7a-c, there are swirl motions at horizontal planes of $5 \mathrm{~mm}$ under TDC, with MVL $6.8 \mathrm{~mm}$ and $1.7 \mathrm{~mm}$ and at different CAs of $120^{\circ} \mathrm{CA}$ (intake valves fully opened), $240{ }^{\circ} \mathrm{CA}$ (intake valves totally closed) and $300{ }^{\circ} \mathrm{CA}$ (late stage of the compression). Those flow fields are ensemble-averaged results of 100 cycles with the methods shows in Equation (2).
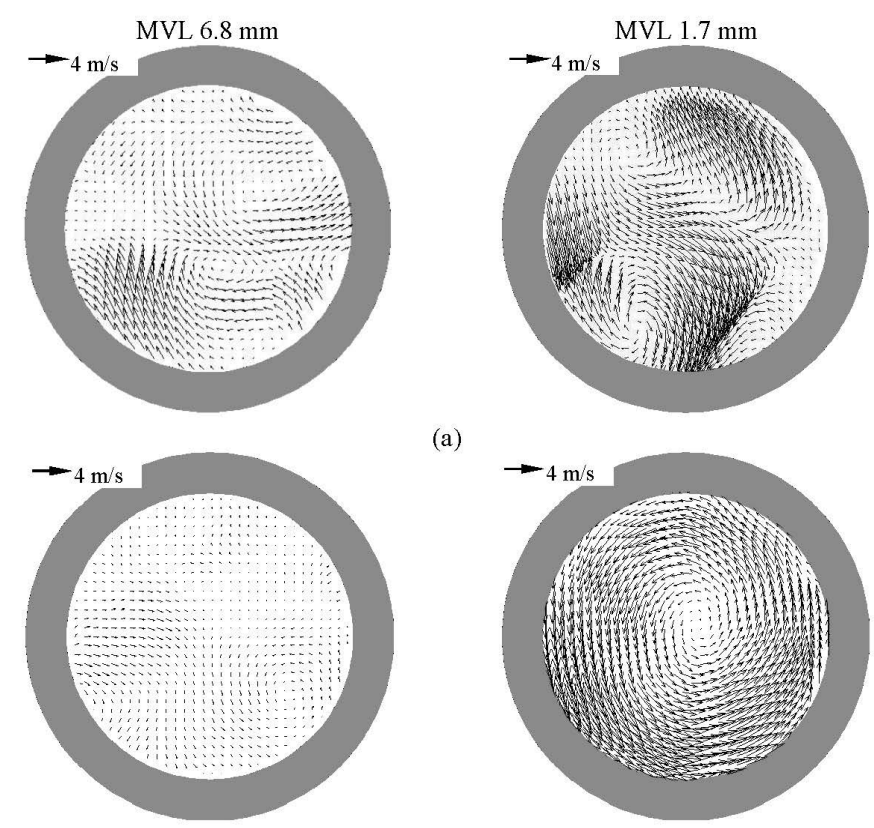

(a)
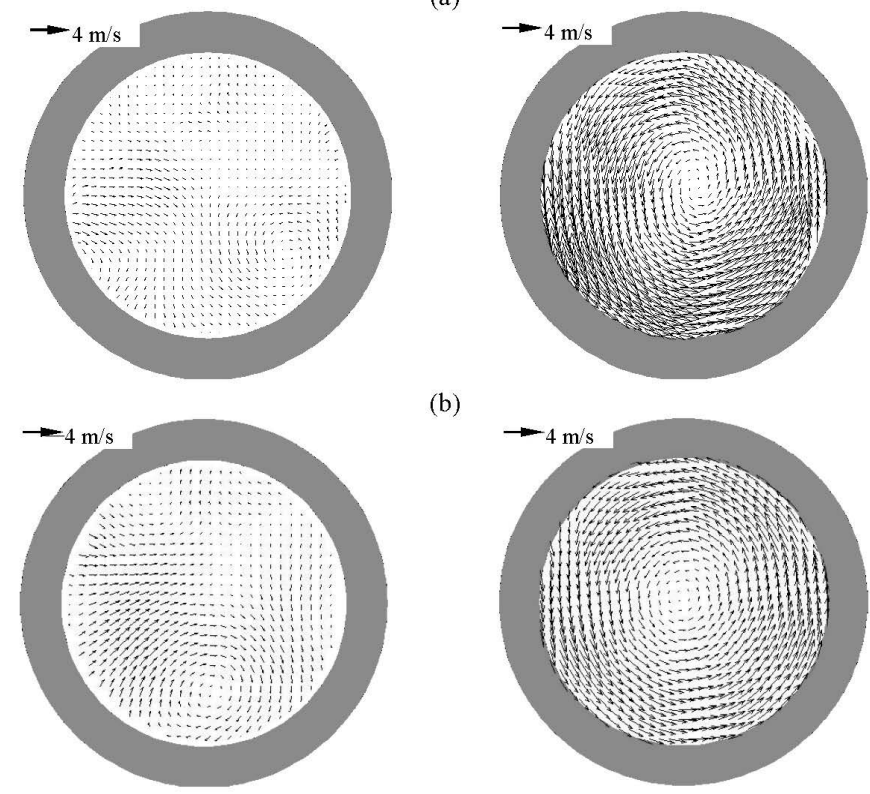

(b)

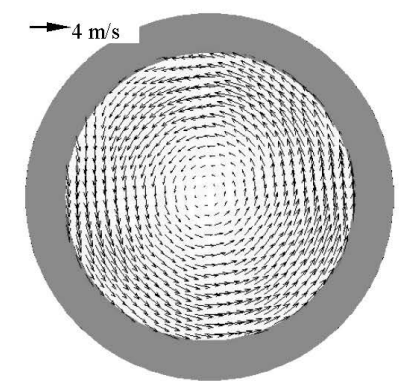

(c)

Figure 7. Swirl flow ensemble-averaged flow fields at the horizontal plane of $5 \mathrm{~mm}$ under TDC for MVL $6.8 \mathrm{~mm}$ and MVL $1.7 \mathrm{~mm}$. (a) At $120{ }^{\circ} \mathrm{CA}$ (close to the maximum valve open); (b) at $240{ }^{\circ} \mathrm{CA}$ (close to the valve close); (c) at $300{ }^{\circ} \mathrm{CA}$ (close to the compression end). CA: crank angle.

Obviously it can be seen from Figure 7a that the intake flow for MVL $1.7 \mathrm{~mm}$ had much higher velocity vectors than that of MVL $6.8 \mathrm{~mm}$ when the intake valves were nearly fully opened. This demonstrates that there was a much higher pressure difference across the intake valves for 
MVL $1.7 \mathrm{~mm}$ due to a smaller curtain area for achieving adequate intake air amount. The high pressure difference and small valve lift result in the flow fields much more irregular for MVL $1.7 \mathrm{~mm}$ than $6.8 \mathrm{~mm}$. For MVL $1.7 \mathrm{~mm}$, there were two vortex centers observed under two intake valves (positions of two intake valves can be found in Figure 4a). This suggested they should be formed from intake flows from two intake valves. For MVL $6.8 \mathrm{~mm}$, though two vortex centers could be also observed, they were very weak and located near exhaust valves rather than intake valves.

With the progress of the intake process, a very strong swirl flow with an apparent vortex center near the cylinder axis could be seen for MVL $1.7 \mathrm{~mm}$ at $240{ }^{\circ} \mathrm{CA}$ when the intake valves would fully close (Figure 7b). For MVL $6.8 \mathrm{~mm}$, the flow field was still dominated by irregular air motion with very weak flow velocities. As it had been the timing when the intake process would finish, the flow strength shown in Figure $7 \mathrm{~b}$ could be considered as the results of the intake process. Those differences of flow field between MVL $1.7 \mathrm{~mm}$ and $6.8 \mathrm{~mm}$ suggests that reduced MVL would not only produce a high flow velocity, but also form a very obvious swirl motion.

Shown in Figure $7 \mathrm{c}$ are swirl motions at $300{ }^{\circ} \mathrm{CA}$ when it was very close to the compression end. Although there is still a very apparent difference between MVL $1.7 \mathrm{~mm}$ and MVL $6.8 \mathrm{~mm}$ as seen in Figure $7 \mathrm{~b}$, it can be seen that the evolutions of the two flow fields have totally different trends. For MVL $1.7 \mathrm{~mm}$, from $240{ }^{\circ} \mathrm{CA}$ to $300{ }^{\circ} \mathrm{CA}$, the strength of swirl motion decreases a little but the flow field becomes even and the vortex center is closer the cylinder center. For MVL $6.8 \mathrm{~mm}$, from very weak swirl motion at $240{ }^{\circ} \mathrm{CA}$, now at $300^{\circ} \mathrm{CA}$ a large-scale vortex appears in the lower part of the flow field and the strength increased somewhat though not very much. The increase may be because the stronger swirl flows were moved upward by the piston from the cylinder bottom. As the in-cylinder air motion at the compression end is always critical for the air-fuel mixing and the flame propagation, enough attention should be paid to the effects of reduced MVL on those flow fields when relevant information is used for optimizing GDI combustion systems.

In Figure 8, variations of swirl ratio with CA for different MVLs at different measured planes are shown. The estimates of swirl ratio were based on those ensemble-averaged velocity fields measured at different CAs and Equation (7) was used for those calculations.

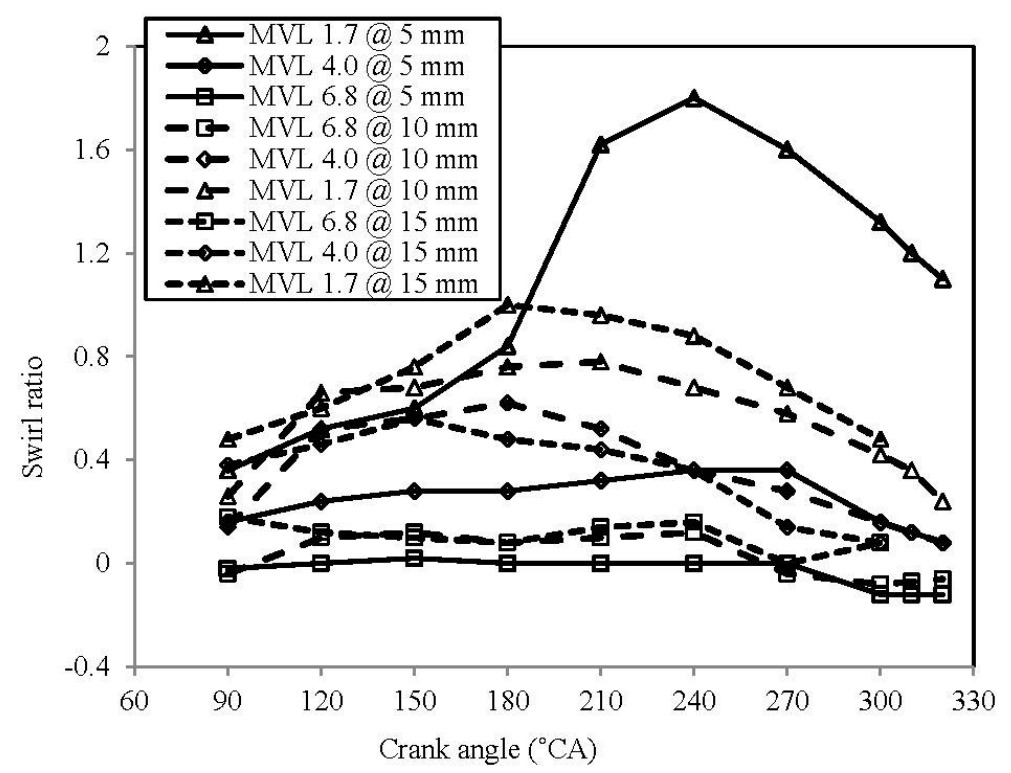

Figure 8. Comparison of swirl ratio variations with $\mathrm{CA}$ at the different measured planes between different MVLs ( $5 \mathrm{~mm}$ - the measured plane of $5 \mathrm{~mm}$ under TDC; $10 \mathrm{~mm}$-the measured plane of 10 $\mathrm{mm}$ under TDC; $15 \mathrm{~mm}$ - the measured plane of $15 \mathrm{~mm}$ under TDC). 
With regard to the $5 \mathrm{~mm}$ plane result, it can be found that the reduced MVLs could result in the increase of swirl ratio at all different CAs. For MVL $6.8 \mathrm{~mm}$, the swirl ratio at this measured plane varies around zero and there might be different rotating directions at different CAs. Before $180^{\circ} \mathrm{CA}$, the reductions of MVL from $4.0 \mathrm{~mm}$ to $1.7 \mathrm{~mm}$ regularly produce a double swirl ratio compared to the MVL reduction from $6.8 \mathrm{~mm}$ to $4.0 \mathrm{~mm}$. From $180^{\circ} \mathrm{CA}$ to $240^{\circ} \mathrm{CA}$, the increase rate of swirl ratio for MVL 1.7 has a significant growth. As these two CAs were at late intake stroke, the sudden increase of swirl ratio might come from the quick increase of intake flow speed due to a continuous increase of pressure difference over the intake valves. These variations resulted in a much higher swirl ratio for MVL $1.7 \mathrm{~mm}$ at the compression end stage than MVL $4.0 \mathrm{~mm}$ and $6.8 \mathrm{~mm}$.

For results at $10 \mathrm{~mm}$ and $15 \mathrm{~mm}$ planes, it can be seen the increase of swirl ratio for reduced MVLs at lower measured planes is still very obvious, but the increase rates are not as high. Similar to those findings shown in Figure 7, the increase of swirl ratio due to reduced MVL is more obvious at near intake valve area or the upper volume of the cylinder.

\subsection{Effects of Maximum Valve Lifts on Fluctuating Kinetic Energy Based on Swirl Flows}

As mentioned in Section 2, the high-frequency fluctuating velocity and the low-frequency fluctuating velocity are produced by totally different sources. In this study, the high-frequency fluctuating velocity refers to the in-cycle turbulence which should be beneficial to the air-fuel mixing and the flame propagation. For extracting different frequency fluctuating kinetic energy as demonstrated in Section 2, it needs to be noted that the variation of the in-cylinder density was not taken into account due to the difficulty in obtaining the density distribution in the cylinder. In the other hand, the actual spatial variation of in-cylinder density is normally small enough to be ignored, in particular for gasoline engines. Those results shown in Figures 9 and 10 are also based on 100 measured cycles.

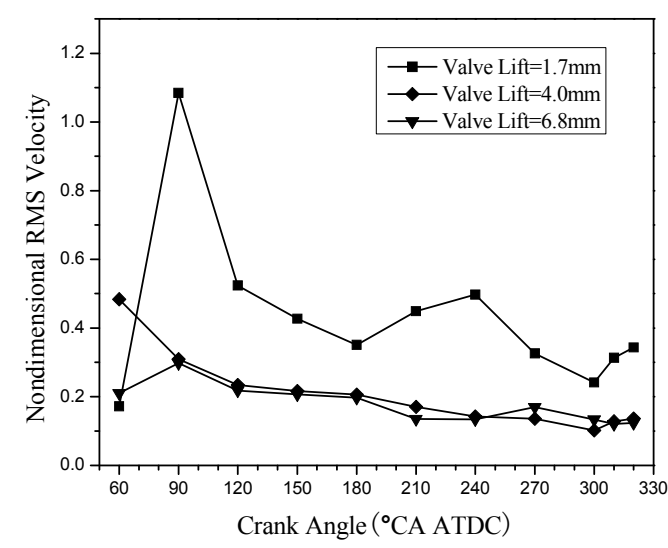

(a)

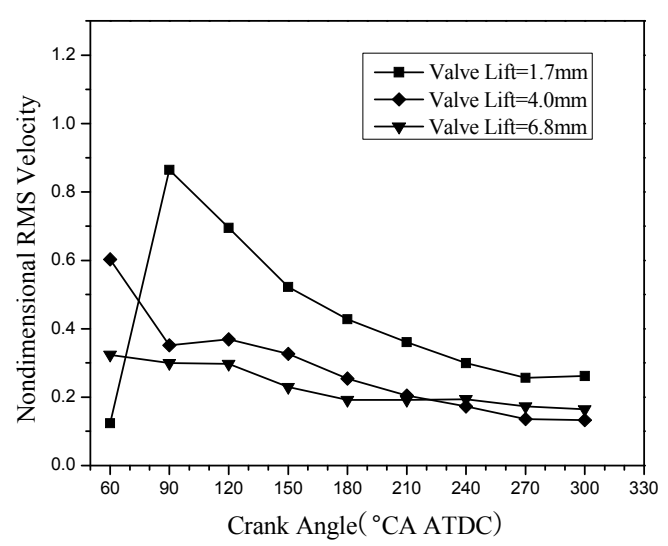

(b)

Figure 9. Variations of the total high-frequency non-dimensional root-mean-square (RMS) fluctuating velocity of swirl flow with CA for three MVLs (at (a) $5 \mathrm{~mm}$ and (b) $15 \mathrm{~mm}$ planes).

As shown in Figure 9, those are variations of total high-frequency non-dimensional RMS fluctuating velocity with CA for different MVLs. As the non-dimensional RMS fluctuating velocity for which the definition has been shown in Section 2 is based on the fluctuating kinetic energy, each value of total high-frequency non-dimensional RMS fluctuating velocity shown in the figure was actually achieved from the total high-frequent fluctuating kinetic energy which is the integration of high frequent fluctuating kinetic energy for all spatial points at the measured plane. From those results, it can be seen that the high-frequency non-dimensional RMS fluctuating velocity keeps decreasing with the CA, due to gradually dissipated small- and micro-scale vortexes. When MVL is reduced from $6.8 \mathrm{~mm}$ to $4.0 \mathrm{~mm}$, the increase of the high-frequency non-dimensional RMS fluctuating velocity 
is not so apparent, but from MVL 4.0 to $1.7 \mathrm{~mm}$, there is a very remarkable increase at all CAs and at different measured planes. Until $320^{\circ} \mathrm{CA}$, MVL $1.7 \mathrm{~mm}$ 's total high-frequency fluctuating kinetic energy still keeps very high level compared to MVL $4.0 \mathrm{~mm}$ and $6.8 \mathrm{~mm}$.

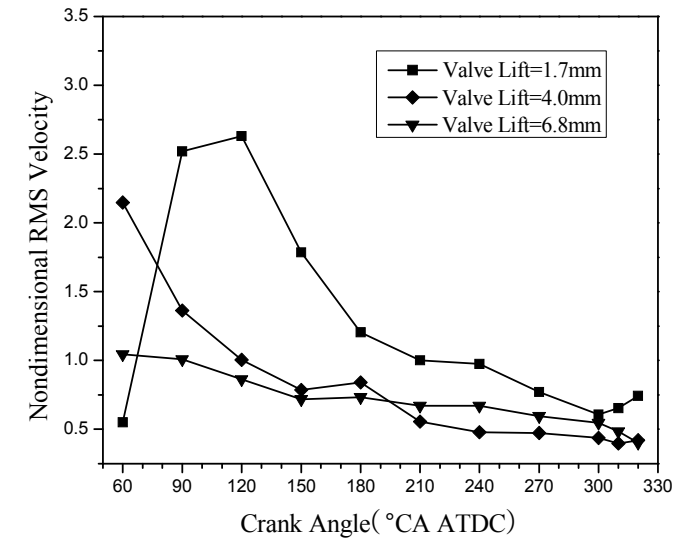

(a)

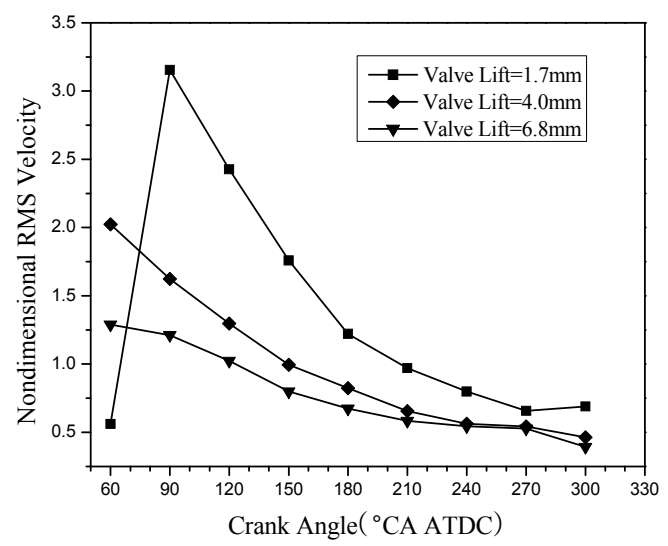

(b)

Figure 10. Variations of total low-frequency non-dimensional RMS fluctuating velocity of swirl flow with CA for three MVLs (at (a) $5 \mathrm{~mm}$ and (b) $15 \mathrm{~mm}$ plane).

While low-frequency fluctuating kinetic energy mainly results from the cyclic variation, its impact on the air-fuel mixing and combustion is not as simple as the impact of high-frequency fluctuating kinetic energy. On the one hand, it may provide useful assistance to the mixing similar as from high-frequency fluctuating kinetic energy but at a different spatial scale. On the other hand, it could possibly be the main reason for causing the cycle-to-cycle variation of the combustion owing to its long spatial scale and high strength relatively. As shown in Figure 10, the variation of total low-frequency non-dimensional RMS velocity with CA has a similar trend to that of total high-frequency non-dimensional RMS velocity, but the increase at the compression end for reduced MVL is not so high, as shown in Figure 10.

In brief, when reduced MVL is used to provide some benefits for gasoline engine combustion and emissions by reducing the pumping losses and improving the air-fuel mixing and flame propagation, special attention should be paid to the possible drawback of increasing cycle-to-cycle variation due to the increase of fluctuating kinetic energy. In addition, only swirl flow details are presented in this paper for the effects of VVL on the in-cylinder air motion. From the results achieved under steady flow tests, it is shown that the reduced MVL has a reverse influence on the tumble flow. Further analysis should be considered for the total effect of VVL on the in-cylinder air motion by combining the presentations of swirl and tumble flows.

\subsection{Effects of Maximum Valve Lifts on Tumble Flow Field and Tumble Ratio}

Figure 11a,b, it show the tumble flow fields under MVLs of $1.7 \mathrm{~mm}, 4.0 \mathrm{~mm}$ and $6.8 \mathrm{~mm}$ at CA of $128{ }^{\circ} \mathrm{CA}$ at which intake valves are fully opened. It needs to be noted that those flow fields are still ensemble-averaged from 100 continuous cycles. One of main features in the figures is that the maximum flow velocity decreases obviously in both planes with the increase of MVL. This may be because the adequate intake air amount for higher MVL during the early intake stage resulted in very small in-cylinder vacuum and low intake flow velocity. For the flow field, it can be found in Figure 11a that with the MVL of $1.7 \mathrm{~mm}$, two oppositely rotating tumble structures exit, and this is presented more clearly in Figure 11b. For the MVL of $4.0 \mathrm{~mm}$, two tumble structures can still be seen, but it becomes there is only an obvious tumble vortex for the MVL of $6.8 \mathrm{~mm}$. This suggests there is a strong interaction between the intake air flow and the cylinder wall under the MVLs of $1.7 \mathrm{~mm}$ and $4.0 \mathrm{~mm}$. When the MVL increases to $6.8 \mathrm{~mm}$, the take air flows toward more to the 
piston top direction. Then it results in the formation of the single rotation direction structure, with a large scale structure.

Although the whole range of the in-cylinder tumble flow can't be imaged because of the limited measurement area, the result from the current measurable region shows that a large scale with the same rotating orientation is more easily formed under high MVL, while small scale counter-clockwise rotating flows exist because of the interaction and obstruction among the flows moving from different sides of the cylinder wall under low MVL conditions.

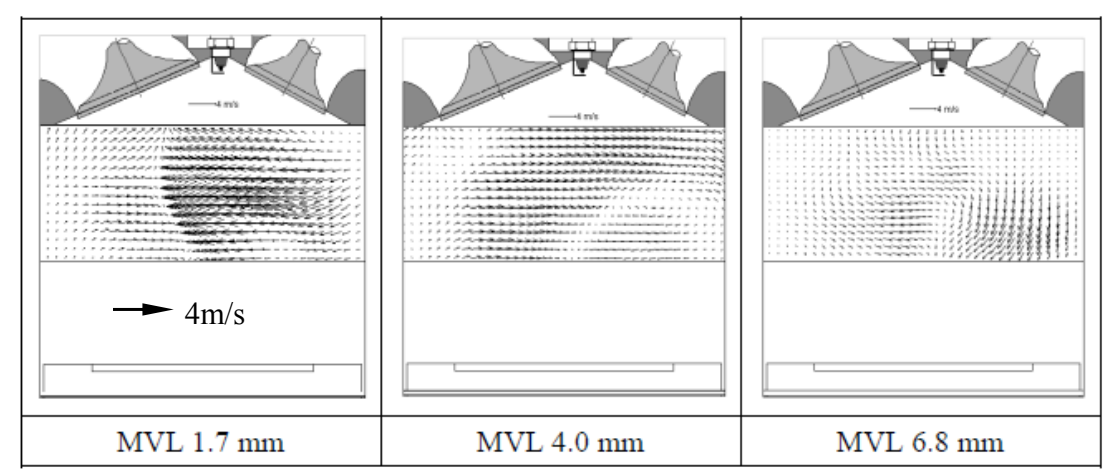

(a)

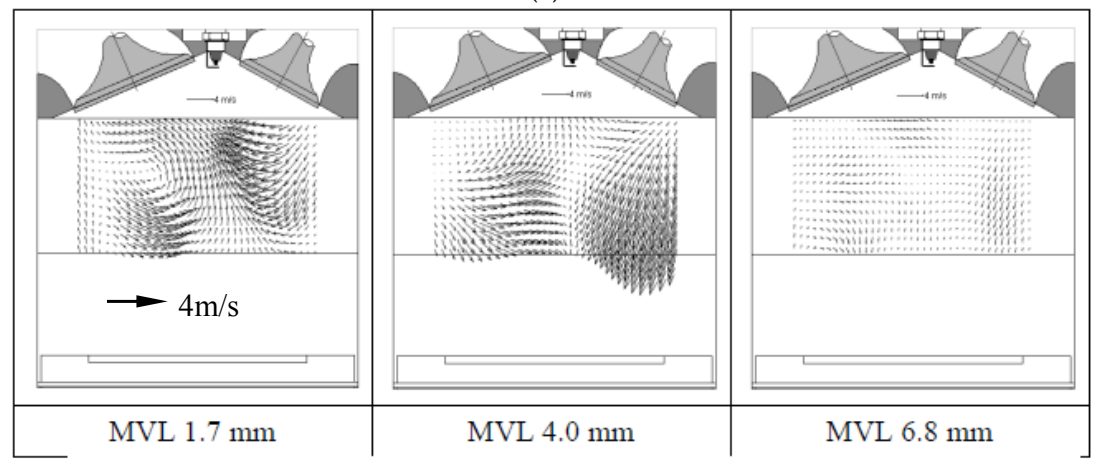

(b)

Figure 11. Tumble flow ensemble-averaged flow fields at $128^{\circ} \mathrm{CA}$ (during the intake stroke) for MVL $1.7 \mathrm{~mm}, 4.0 \mathrm{~mm}$ and $6.8 \mathrm{~mm}$ : (a) at Plane 1 and (b) at Plane 2.

Figure 12a,b shows the ensemble-averaged flow fields in two measurement planes with MVLs of $1.7 \mathrm{~mm}, 4.0 \mathrm{~mm}$ and $6.8 \mathrm{~mm}$ when the intake valves are completely closed ( $\left.228^{\circ} \mathrm{CA}\right)$. It can be found in Figure $7 \mathrm{a}$ that distortion and break happen in the tumble structure owing to the compression. For the MVL of $1.7 \mathrm{~mm}$, the clockwise tumble structure on the left side of the cylinder has decayed. The flow motions at two planes present similar flow structure and a clockwise large scale tumble structure is dominant in the flow field, and the maximum velocity is approximately $7 \mathrm{~m} / \mathrm{s}$. For the MVL of $6.8 \mathrm{~mm}$, it can be found the tumble structure has broken in the measurement plane and no obvious vortex can be found. At Plane 2 (directly under valves) in Figure 12b, velocity trends have apparently become weak while still lower MVLs still have high velocity vectors.

When the piston moves to near the end of the compression stroke, as shown in Figure 13 (at $300{ }^{\circ} \mathrm{CA}$ ), the obvious big vortexes for the MVLs of $1.7 \mathrm{~mm}$ and $4 \mathrm{~mm}$ have disappeared in the measurement area, but an obvious vortex exists for MVL of $6.8 \mathrm{~mm}$. The reason may be that the main tumble flow energy of the MVL of $6.8 \mathrm{~mm}$ may exit in the cylinder bottom area which can't be seen in the observed area at $128{ }^{\circ} \mathrm{CA}$ and $228^{\circ} \mathrm{CA}$. This should be due to stronger vertical intake flow velocity of the MVL of $6.8 \mathrm{~mm}$. 


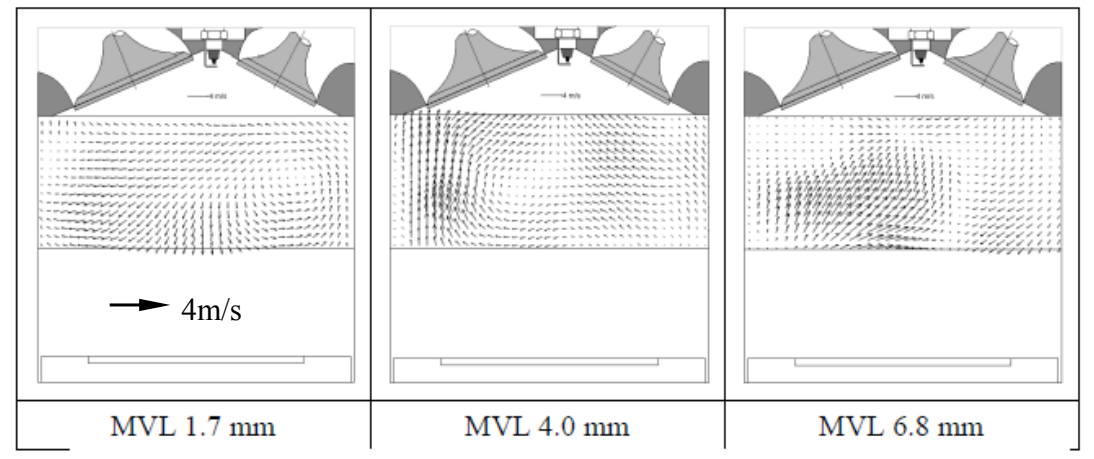

(a)

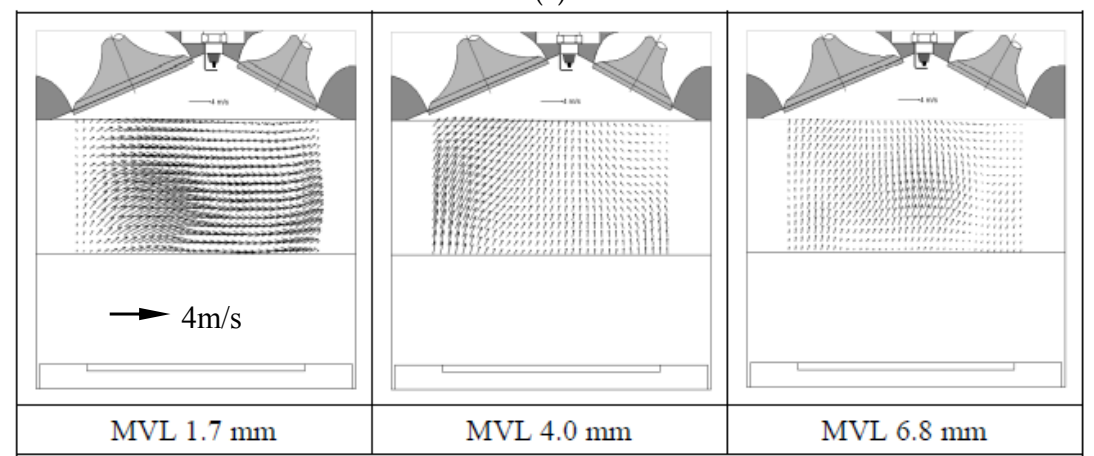

(b)

Figure 12. Tumble flow ensemble-averaged flow fields at $228{ }^{\circ} \mathrm{CA}$ (during compression stroke) for MVL $1.7 \mathrm{~mm}, 4.0 \mathrm{~mm}$ and $6.8 \mathrm{~mm}$ : (a) at Plane 1 and (b) at Plane 2.

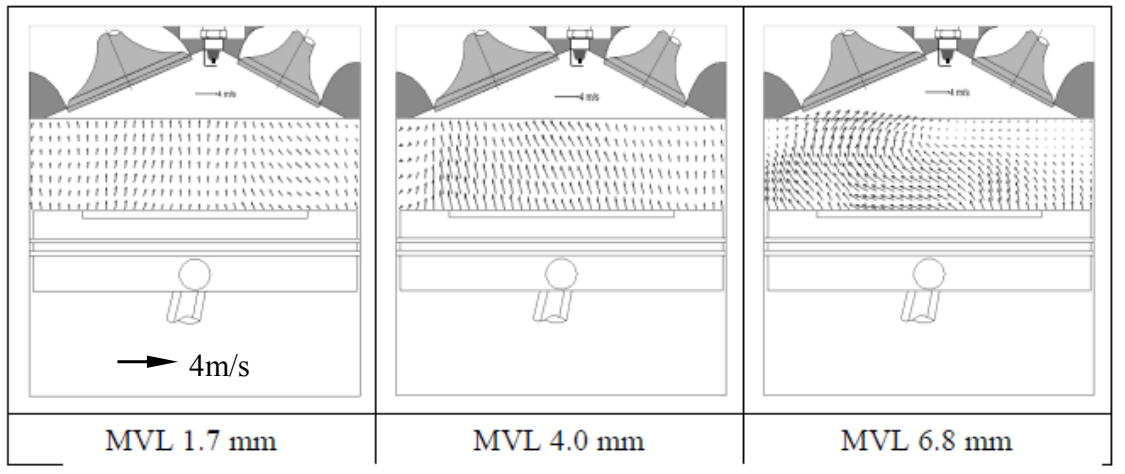

(a)

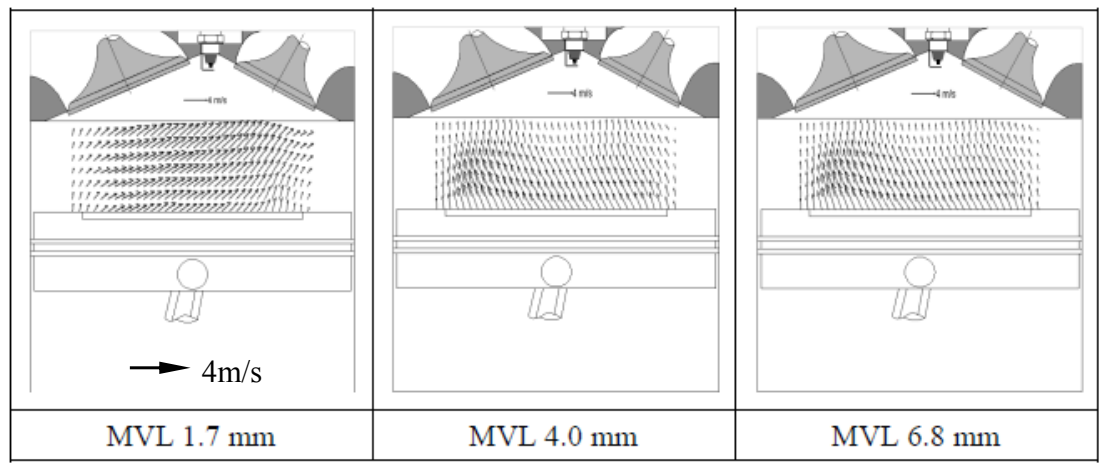

(b)

Figure 13. Tumble flow ensemble-averaged flow fields at $300{ }^{\circ} \mathrm{CA}$ (near the compression end) for MVL $1.7 \mathrm{~mm}, 4.0 \mathrm{~mm}$ and $6.8 \mathrm{~mm}$ : (a) at Plane 1 and (b) at Plane 2. 
Figure 14a,b shows the variations of tumble ratio as the function of CA. As is shown in Figure $14 \mathrm{a}$, between the $\mathrm{CA}$ of $128{ }^{\circ} \mathrm{CA}$ and $180{ }^{\circ} \mathrm{CA}$ which is during the intake stroke, the counter-clockwise rotating flow takes the predominant place in the measurement plane for the MVL of $1.7 \mathrm{~mm}$, then high positive tumble ratio values are produced. For MVLs of $4.0 \mathrm{~mm}$ and $6.8 \mathrm{~mm}$, lower tumble ratio with negative values are presented due to the intake air flows' domination at clockwise direction. Between $120{ }^{\circ} \mathrm{CA}$ and $210{ }^{\circ} \mathrm{CA}$, the MVL of $4.0 \mathrm{~mm}$ and the MVL of $6.8 \mathrm{~mm}$ produced very similar tumble ratios (direction and value), but during the compression stroke, the tumble ratio of the MVL of $4.0 \mathrm{~mm}$ decayed quickly, while the tumble of $6.8 \mathrm{~mm}$ MVL kept the direction and strength very well. This should still come from the reason as mentioned in the last section-the more tumble flow energy of the MVL of $6.8 \mathrm{~mm}$ stays just above the piston top. Similarly the tumble ratio of $1.7 \mathrm{~mm}$ MVL becomes weak very quickly during the compression stroke due to unapparent vortex structure, though most of velocity vectors are still big in the measurement area.

Figure 14b reveals that a counter-clockwise flow structure dominates the flow for the MVL of $1.7 \mathrm{~mm}$ at the early intake stroke, as the piston moves to the bottom dead centre (BDC), clockwise tumble structure predominate in the plane near the BDC $\left(150^{\circ} \mathrm{CA}\right.$ to $\left.180^{\circ} \mathrm{CA}\right)$ until the end of the compression of the compression stroke. For the MVL of $6.8 \mathrm{~mm}$, as the air intake in the early stage of the intake stroke has made the in-cylinder vacuum so small, the intake flow velocity is very low and this results in the very low tumble ratio (in the measurement area).

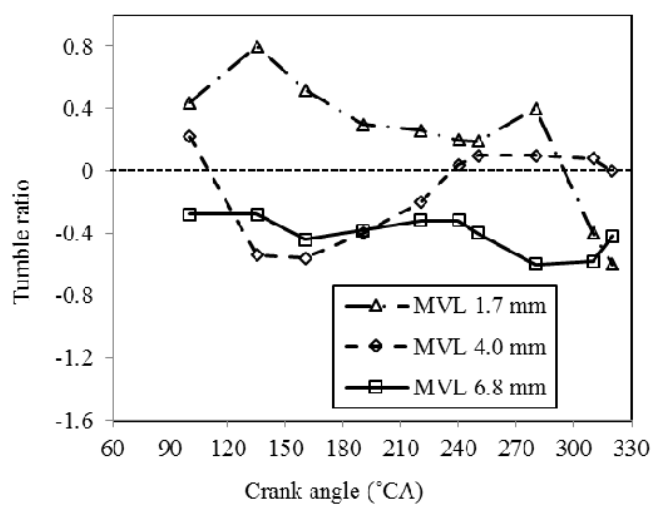

(a)

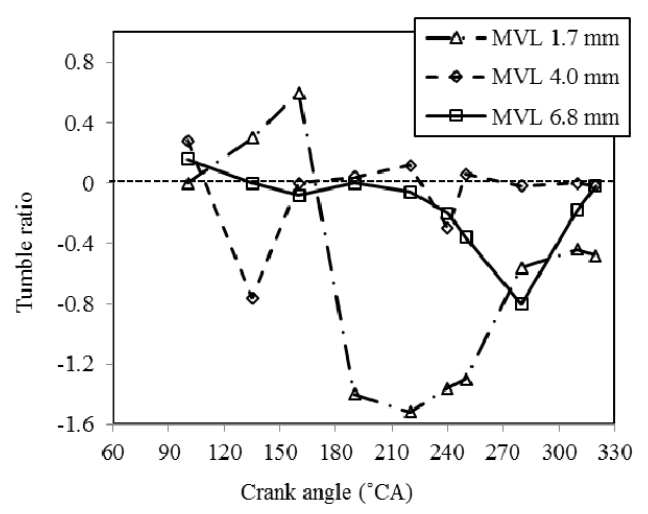

(b)

Figure 14. Tumble ratio varations as function of CA: (a) at Plane 1 and (b) at Plane 2.

Figure 14a indicates at the end of compression stoke, the modulus of the tumble ratio with the MVL of $6.8 \mathrm{~mm}$ can be up to 0.6 and is much larger than that of other two MVLs. For GDI engines which have fuel injection around this time, this is especially beneficial for fuel-air mixing. This suggests propitious in-cylinder tumble motion can be provided for GDI fuel-air mixing when higher valve lift is operated.

\subsection{Effects of Maximum Valve Lifts on Fluctuating Kinetic Energy based on Tumble Flows}

As shown in Figure 15a,b, there are the velocity kinetic energy variations as a function of the CA for the MVL of $1.7 \mathrm{~mm}$ and $6.8 \mathrm{~mm}$. For each MVL, the results shown comprise the total kinetic energy, the total fluctuation kinetic energy, the high-frequency fluctuation kinetic energy and low-frequency fluctuation kinetic energy. Compared to the MVL of $1.7 \mathrm{~mm}$, all those four kinetic parameters of $6.8 \mathrm{~mm}$ MVL have much smaller valves at all CA positions, though as demonstrated in last section, the MVL of $6.8 \mathrm{~mm}$ produced a higher tumble ratio than the MVL of $1.7 \mathrm{~mm}$ at the compression end. 


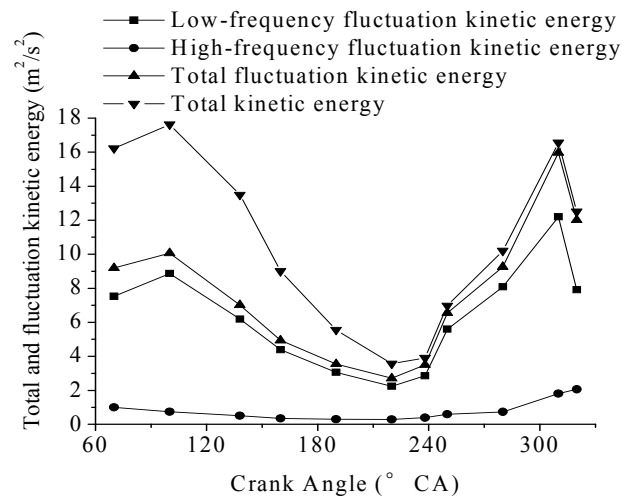

(a)

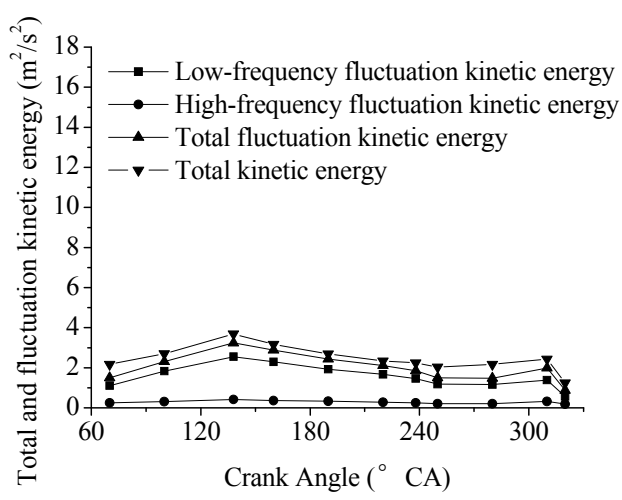

(b)

Figure 15. Averaged fluctuation kinetic energy of 100 cycles at Plane 1. (a) $\mathrm{MVL}=1.7 \mathrm{~mm}$; (b) $\mathrm{MVL}=$ $6.8 \mathrm{~mm}$.

From the results of both the MVL of $1.7 \mathrm{~mm}$ and $6.8 \mathrm{~mm}$, it can be found that the kinetic energy fluctuation is dominant in the total velocity energy. Furthermore, the low frequency fluctuation energy accounted for about $90 \%$ of the total fluctuation kinetic energy. The proportion of fluctuation kinetic energy to the total velocity energy is higher than that obtained by Druault et al. [25], and some of the reason is that the engine speed was set lower in the present study, which may enhance the velocity fluctuation [26]. Moreover, the energies of the flow fields were enhanced largely as the MVL was decreased, which can be seen by comparing the same kind of energy curves at the different MVL conditions. The total energy at lower MVL conditions was about four times more than those at the higher MVL conditions and the CA at which the peak reached was advanced by about $30^{\circ} \mathrm{CA}$.

Combining the results of swirl flow and tumble flow, demonstrates the reduced MVL can more or less improve air-fuel mixing and flame propagation due to enhanced high-frequency fluctuation kinetic energy, but simultaneously it may increase cycle-to-cycle variations due to increased low-frequency fluctuation kinetic energy.

\section{Conclusions}

An investigation into in-cylinder swirl flow and tumble flow under reduced MVL has been carried out on an optical engine. A PIV system including necessary data analysis has been applied for examining swirl and tumble flow field, swirl and tumble ratio variation and fluctuating kinetic energy distribution. The following conclusions have been drawn from the present study:

- Initial experiments conducted on a steady flow test rig showed that reduced valve lift would significantly enhance the total swirl strength, but reduce the total tumble ratio.

- Under the present measurement conditions, PIV investigation results showed that reduced MVL could enhance swirl flow velocity, which resulted in a very regular swirl motion in the late stage of the intake process and the strong swirl flow can maintain very well until the late compression stage. However, measurement results also showed that effects of reduced MVL on swirl motion have a stronger presence in the upper part of the cylinder volume than in the bottom part. By comparing swirl ratio for different MVL values, it can be found that reduced MLV can significantly increase swirl ratio at all almost measured CAs and on different measured planes.

- The reduction of MVL can increase both high-frequency and low-frequency swirl flow thus fluctuating the kinetic energy remarkably. In particular at the late stage of the compression process, the increase of high-frequency fluctuating kinetic energy across all spatial points on the measured planes will be beneficial to air-fuel mixing, but the simultaneous increase of low-frequency fluctuating kinetic energy will possibly result in high combustion cyclic variation. 
- Regarding tumble flow, the ensemble-averaged flow field results demonstrate that lower MVLs result in more horizontal intake flow velocity vectors which can be easily detected under the valve seat area, while higher MVLs can produce more vertical flows which turn more toward to the piston top and finally are more possible to form big scale tumble flow structure.

- Although the result of lower MVLs show a higher tumble ratio when the measuring range can't cover most cylinder area, higher MVLs substantially produce higher tumble ratios which can be confirmed when most of the cylinder area lies in the measuring range with the pistons moving close to TDC. The big scale vortex structures produced by higher MVLs made main contribution for producing high tumble ratios of higher MVLs.

- In terms of kinetic energy tumble flow, lower MVLs result in higher values due to higher velocity vectors, though most of the time they did not form good big vortex structures. Based on higher total kinetic energy under lower MVL condition, higher fluctuating energy including high-frequency and low-frequency fractions are presented under lower MVL conditions. The result would on the one hand be helpful for better air-fuel mixing and flame propagation, but on the other hand it would enhance cyclic variations.

Acknowledgments: The financial supports from NSFC (50876074) and the Ministry of Education of China via 985 Program are gratefully acknowledged.

Author Contributions: The investigation was leaded and supervised by Tianyou Wang and Zhijun Peng. Experimental works, data processing and most illustrations were completed by Daming Liu, Gangde Wang and Bingqian Tan. The manuscript was finalized by Zhijun Peng.

Conflicts of Interest: The authors declare no conflict of interest.

\section{Abbreviations}

$\begin{array}{ll}\text { ABDC } & \text { After bottom dead centre } \\ \text { ATDC } & \text { After top dead centre } \\ \text { BBDC } & \text { Before bottom dead centre } \\ \text { BDC } & \text { Bottom dead centre } \\ \text { BTDC } & \text { Before top dead centre } \\ \text { CA } & \text { Crank angle } \\ \text { CAI } & \text { Controlled auto-ignition } \\ \text { CCD } & \text { Charge coupled device } \\ \text { EIVC } & \text { Early intake valve close } \\ \text { EVC } & \text { Exhaust valve close } \\ \text { EVO } & \text { Exhaust valve open } \\ \text { FFT } & \text { Fast Fourier transform } \\ \text { GDI } & \text { Gasoline direct injection } \\ \text { IVC } & \text { Intake valve close } \\ \text { IVO } & \text { Intake valve open } \\ \text { LIVC } & \text { Late intake valve close } \\ \text { LIVO } & \text { Late intake valve open } \\ \text { MVL } & \text { Maximum valve lift } \\ \text { PFI } & \text { Port fuel injection } \\ \text { PIV } & \text { Particle image velocimetry } \\ \text { PSD } & \text { Power spectral density } \\ \text { RMS } & \text { Root mean square } \\ \text { TDC } & \text { Top dead centre } \\ \text { TMVL } & \text { Twin mechanical variable lift } \\ \text { VVA } & \text { Variable valve actuation } \\ \text { VVL } & \text { Variable valve lift } \\ \text { VVO } & \text { Variable valve overlap } \\ \text { VVT } & \text { Variable valve timing } \\ & \end{array}$




\section{References}

1. Stan, C.; Stanciu, A.; Troeger, R.; Mueller, D. GDI Compact Four Stroke Engine-An Advanced Concept for Vehicle Application. In Proceedings of the 2004 SAE World Congress, Detroit, MI, USA, 8-11 March 2004.

2. Lee, K.; Lee, C. An Experimental Study of the Extent of the Operating Region and Emission Characteristics of Stratified Combustion Using the Controlled Autoignition Method. Energy Fuels 2006, 20, 1862-1869. [CrossRef]

3. Hong, H.; Parvate-Patil, G.B.; Gordon, B. Review and analysis of variable valve timing strategies-Eight ways to approach. Proc. Inst. Mech. Eng. 2004, 218, 1179-1200. [CrossRef]

4. Fernandez, H.; Kazour, Y.; Knauf, M.; Sinnamon, J.; Suh, E.; Glueck, D. Development of Continuously Variable Valve Lift Mechanism for Improved Fuel Economy. In Proceedings of the 2012 SAE World Congress, Detroit, MI, USA, 24-26 April 2012.

5. Chen, L.; Stone, R. Measurement of enthalpies of vaporization of isooctane and ethanol blends and their effects on PM emissions from a GDI engine. Energy Fuels 2011, 25, 1254-1259. [CrossRef]

6. Gray, C. A Review of Variable Engine Valve Timing. In Proceedings of the 1988 SAE World Congress, Detroit, MI, USA, 29 February-4 March 1988.

7. Flierl, R.; Gollasch, D.; Knecht, A.; Hannibal, W. Improvements to a Four Cylinder Gasoline Engine through the Fully Variable Valve Lift and Timing System UniValveß. In Proceedings of the 2006 SAE World Congress, Detroit, MI, USA, 3-6 April 2006.

8. Stansfield, P.A.; Wigley, G.; Garner, C.; Patel, R. Unthrottled Engine Operation using Variable Valve Actuation: The Impact on the Flow Field, Mixing and Combustion. In Proceedings of the 2007 SAE World Congress, Detroit, MI, USA, 16-19 April 2007.

9. Grimaldi, F.; Gervais, D.; Marchal, A.; Floch, A. Single-cylinder Experiments for Downsizing-Oriented SI Concepts: GDI and VVL Thermodynamic Comparison. In Proceedings of the 2004 SAE World Congress, Detroit, MI, USA, 8-11 March 2004.

10. Kiyoshi, H.; Kazumasa, L.; Hirohumi, H. Development of a New Multi-Mode Variable Valve Timing Engine. In Proceedings of the 1993 SAE World Congress, Detroit, MI, USA, 1-5 March 1993.

11. Flierl, R.; Klüting, M. The Third Generation of Valvetrains-New Fully Variable Valvetrains for Throttle-Free Load Control. In Proceedings of the 2000 SAE World Congress, Detroit, MI, USA, 6-9 March 2000.

12. Stokes, J.; Lake, T.H.; Murphy, R.D. Gasoline Engine Operation with Twin Mechanical Variable Lift (TMVL) Valvetrain Stage 1: SI and CAI Combustion with Port Fuel Injection. In Proceedings of the 2005 SAE World Congress, Detroit, MI, USA, 11-14 April 2005.

13. Sellnau, M.; Kunz, T.; Sinnamon, J.; Burkhard, J. 2-Step Variable Valve Actuation: System Optimization and Integration on an SI Engine. In Proceedings of the 2006 SAE World Congress, Detroit, MI, USA, 3-6 April 2006.

14. Cleary, D.; Silvas, G. Unthrottled Engine Operation with Variable Intake Valve Lift, Duration, and Timing. In Proceedings of the 2007 SAE World Congress, Detroit, MI, USA, 16-19 April 2007.

15. Fischer, J.; Velji, A.; Spicher, U. Investigation of Cycle-to-Cycle Variations of In-Cylinder Processes in Gasoline Direct Injection Engines Operating With Variable Tumble Systems. In Proceedings of the 2004 SAE World Congress, Detroit, MI, USA, 8-11 March 2004.

16. Hasse, C.; Sohm, V.; Durst, B. Numerical investigation of cyclic variations in gasoline engines using a hybrid URANS/LES modeling approach. Comput. Fluids 2010, 39, 25-48. [CrossRef]

17. Vu, T.T.; Guibert, P. Proper orthogonal decomposition analysis for cycle-to-cycle variations of engine flow. Effect of a control device in an inlet pipe. Exp. Fluids 2012, 52, 1519-1532.

18. Towers, D.P.; Towers, C.E. Cyclic variability measurements of in-cylinder engine flows using high speed particle image velocimetry. Meas. Sci. Technol. 2004, 15, 1917-1925. [CrossRef]

19. Cosadia, I.; Borée, J.; Charnay, G.; Dumont, P. Cyclic variations of the swirling flow in a diesel transparent engine. Exp. Fluids 2006, 41, 115-134. [CrossRef]

20. Dimitriou, P.; Wang, W.; Peng, Z. A piston geometry and nozzle spray angle investigation in a DI diesel engine by quantifying the air-fuel mixture. Int. J. Spray Combust. Dyn. 2015, 7, 1-24. [CrossRef]

21. Lavision FluidMaster. Available online: http://www.lavision.de/en/products/fluidmaster.php (accessed on 15 November 2015). 
22. Li, Y.; Zhao, H.; Peng, Z.; Ladommatos, N. Particle image velocimetry measurement of in-cylinder flow in internal combustion engines-Experiment and flow structure analysis. Proc. Inst. Mech. Eng. D 2002, 216, 65-81. [CrossRef]

23. Miles, P.; Megerle, M.; Hammer, J.; Nagel, Z.; Reitz, R.D.; Sick, V. Late-Cycle Turbulence Generation in Swirl-Supported, Direct-Injection Diesel Engines. In Proceedings of the 2002 SAE World Congress, Detroit, MI, USA, 4-7 March 2002.

24. Reuss, D.L. Cyclic Variability of Large-Scale Turbulent Structures in Directed and Undirected IC Engine Flows. In Proceedings of the 2000 SAE World Congress, Detroit, MI, USA, 6-9 March 2000.

25. Druault, P.; Guibert, P.; Alizon, F. Use of proper orthogonal decomposition for time interpolation from PIV data Application to the cycle-to-cycle variation analysis of in-cylinder engine flows. Exp. Fluids 2005, 39, 1009-1023. [CrossRef]

26. Müller, S.H.R.; Böhm, B.; Gleißner, M. Flow field measurements in an optically accessible, direct-injection spray-guided internal combustion engine using high-speed PIV. Exp. Fluids 2010, 48, 281-290. [CrossRef]

(C) 2015 by the authors; licensee MDPI, Basel, Switzerland. This article is an open access article distributed under the terms and conditions of the Creative Commons by Attribution (CC-BY) license (http:/ / creativecommons.org/licenses/by/4.0/). 